\title{
Maçka (Trabzon) İlçesi ve Çevresinin Otbiçen (Arachnida: Opiliones) Faunası
}

\author{
Kemal KURT ${ }^{*}$ \\ Gümüşhane Üniversitesi, Şiran Mustafa Beyaz Meslek Yüksekokulu, 29700, Şiran/Gümüşhane \\ Geliş tarihi/Received 27.04.2015 \\ Düzeltilerek geliș tarihi/Received in revised form 15.07.2015 \\ Kabul tarihi/Accepted 15.07.2015
}

\begin{abstract}
$\ddot{O}_{z e t}$
Bu çalışmada, Maçka (Trabzon) ve çevresinden 2013 yılının Haziran ayından başlayarak 2014 yılının Eylül ayına kadar yapılan arazi çalışmaları sonucunda toplanan örnekler sistematik açıdan değerlendirildi. Bu değerlendirme sonucunda 4 familya ait toplam 19 takson tespit edildi. Bu taksonlar: Dicranolasma giljarovi Šilhavý, 1966; Giljarovia tenebricosa Redikorzev, 1936; Histricostoma caucasicum (Redikorzev, 1936); Mitostoma gracile (Redikorzev, 1936); Paranemastoma sp.; Vestiferum alatum Martens, 2006; Vestiferum funebre (Redikorzev, 1936); Lacinius erinaceus Staręga, 1966; Mitopus morio (Fabricius, 1779); Odiellus zecariensis Mcheidze 1952; Opilio hemseni Roewer, 1952; Opilio parietinus (De Geer, 1778); Phalangium opilio Linnaeus, 1761; Rafalskia olympica olympica (Kulczyński, 1903); Rilaena ermani Kurt, 2015; Rilaena zakatalica Snegovaya, Chemeris, 2005; Rilaena triangularis (Herbst, 1799); Zachaeus crista (Brullé, 1832); Nelima pontica Charitonov, 1941 araştırma alanından ilk kez kaydedildi. Bu türlerin dünya ve Türkiye'deki dă̆llımları verildi.
\end{abstract}

Anahtar Kelimeler: Fauna, Maçka (Trabzon), Opiliones, Otbiçen, Sistematik, Türkiye

\section{Harvestmen (Arachnida: Opiliones) Fauna of Surrounding Maçka (Trabzon) District}

\begin{abstract}
In this study, the samples collected from the land studies, between June 2013 and September 2014 in Maçka (Trabzon) district, were evaluated systematically. As a result of these evaluations, 19 species belonging to 4 families were determined. These taxa: Dicranolasma giljarovi Šilhavý, 1966; Giljarovia tenebricosa Redikorzev, 1936; Histricostoma caucasicum (Redikorzev, 1936); Mitostoma gracile (Redikorzev, 1936); Paranemastoma sp.; Vestiferum alatum Martens, 2006; Vestiferum funebre (Redikorzev, 1936); Lacinius erinaceus Starega, 1966; Mitopus morio (Fabricius, 1779); Odiellus zecariensis Mcheidze 1952; Opilio hemseni Roewer, 1952; Opilio parietinus (De Geer, 1778); Phalangium opilio Linnaeus, 1761; Rafalskia olympica olympica (Kulczyński, 1903); Rilaena ermani Kurt, 2015; Rilaena zakatalica Snegovaya, Chemeris, 2005; Rilaena triangularis (Herbst, 1799); Zachaeus crista (Brullé, 1832); Nelima pontica Charitonov, 1941 were recorded in the study area for the first time. This species was given distribution in the world and Turkey.
\end{abstract}

Keywords: Fauna, Harvestmen, Maçka (Trabzon), Opiliones, Systematics, Turkey

\footnotetext{
*Kemal KURT, kemalkurtmyo@gmail.com, Tel: (0456) 2331032
} 


\section{Giriş}

Otbiçenler (Opiliones), Eklembacaklılar (Arthropoda) şubesinin örümceğimsiler (Arachnida) sınıfı içerisinde yer alan bir takım olup tür sayısı bakımından örümcek ve kenelerden sonra üçüncü büyük takımını oluşturur. Otbiçenler yaklaşık olarak ülkemizde 100, Avrupada 310, Palearktik bölgede 801, dünyada ise 6125 takson ile temsil edilmektedirler (Mitov, 2007; Kury, 2012, 2013; Kurt 2014).

Avrupa'da birçok ülkenin otbiçen biyoçeşitliliği tam olarak tespit edilmesine rağmen, ülkemizde otbiçenler üzerine yeterince çalışma yapılmamıştır. Ülkemiz otbiçenleri üzerine ilk çalışmalar yabancı bilim insanları tarafindan kapsamlı olmayan faunistik araştırmalar şeklinde gerçekleştirilmiştir. Son y1llarda otbiçenler üzerine ülkemiz araştırmacıları tarafından yapılan çalışmalar hızla artmaktadır. Yerli araştırmacılar tarafından yapılan ilk çalışmalar yüksek lisans ve doktora tez çalışmalarıdır. Günümüzde ise gerek faunistik gerekse sistematik araştırmalar hızla devam etmektedir. İran, Suriye, Irak ve İsrail gibi ülkemize yakın bölgelerde otbiçenler üzerine çok az sayıda çalışma yapılmıştır. Azerbaycan, Bulgaristan, Kafkaslar ve Rusya'nın otbiçen faunası üzerine çok sayıda çalışma mevcuttur (Kurt, 2010).

Otbiçenler toprak içerisinde ve yüzeyinde, taş ve ağaç kabuklarının altında, kaya, ağaç ve bitkilerin üzerinde, döküntü içlerinde yaşamsal aktivitelerinin sürdürürler. Otbiçenler, karada yaşayan başta böcekler olmak üzere diğer birçok eklembacaklının etkili predatörü olarak bilinirler (Çorak, 2010).

$\mathrm{Bu}$ grup için dünyada farklı isimler kullanılmaktadır. Örneğin çoban örümcekleri anlamına gelen "shepherd spiders" terimi kullanılmıştır. Bazı Avrupa ülkelerinde çobanlar sürüyü daha iyi kontrol etmek için uzun çubuklar üzerinde yürürler. Otbiçenlerin hareketleri Avrupa'daki çobanların yürüyüşüne benzetildiği için bu terim kullanılmıştır (Hillyard ve Sankey 1990). Ayrica bu grubun üyelerinin hareketi orakla ot biçen bir çiftçiye benzediğinden dolayı bu grup için "otbiçen" terimi kullanılmıştır (Bayram, vd., 2007).

Otbiçenler vücutlarının tek parça olması, sefalotoraks ve abdomenin tüm genişliğince birleşmesi, abdomenin segmentli yapıda olması, bir çift gözlerinin bulunması, koku bezlerine sahip olması, zehir ve a $\breve{g}$ bezlerinin bulunmaması gibi özelliklerinden dolayı diğer araknid gruplarından kolayca ayırt edilirler (Hillyard ve Sankey, 1990).

Araştırma alanın florasının bir kısmı araştırılmıştır, fakat faunası ile ilgili önemli bir çalışma yoktur. Bunun yanı sıra bölgenin otbiçen faunası üzerine herhangi bir çalışma yapılmamıștır. Bu alanın faunasını daha net ortaya koymak için otbiçenler yönünden de araştırılması gerekmektedir. Trabzon iline bağlı Maçka ilçesi ve çevresinin otbiçen faunasının belirlenmesi ülkemiz otbiçen faunasının belirlenmesine önemli ölçüde katkı sağlayacaktır.

\section{Gereç ve Yöntem}

Araştırma alanı Maçka ilçesi ve çevresinden oluşmaktadır. Araştırma alanı Sümela Manastırı, Altındere Milli Parkı, Meryemana vadisi, Vazelon ve Kuştul Manastırları ve 137 yaylaya sahip olup, doğusunda Yomra İlçesi, batısında Akçaabat, Düzköy ve Tonya İlçeleri, kuzeyinde Trabzon, güneyinde Gümüşhane İli ve Torul İlçesi bulunmaktadır (Şekil 1). Maçka ilçesi ve çevresi çok değişik habitat ve mikroklima özellikleri içermektedir. $\mathrm{Bu}$ tür özellikler yapılacak olan çalışmayı gerek faunistik gerekse ekolojik açıdan değerli kılmaktadır.

Maçka ve çevresinden 2013 yılı Haziran2014 yılı Eylül ayları arasında belirlenen istasyonlardan aspiratör, çukur tuzak, atrap ve pens yardımıyla otbiçen örnekleri toplanılmıştır. Toplanan örnekler, içerisinde \% 70'lik etil alkol bulunan saklama şişelerine alınarak etiketlendi. 


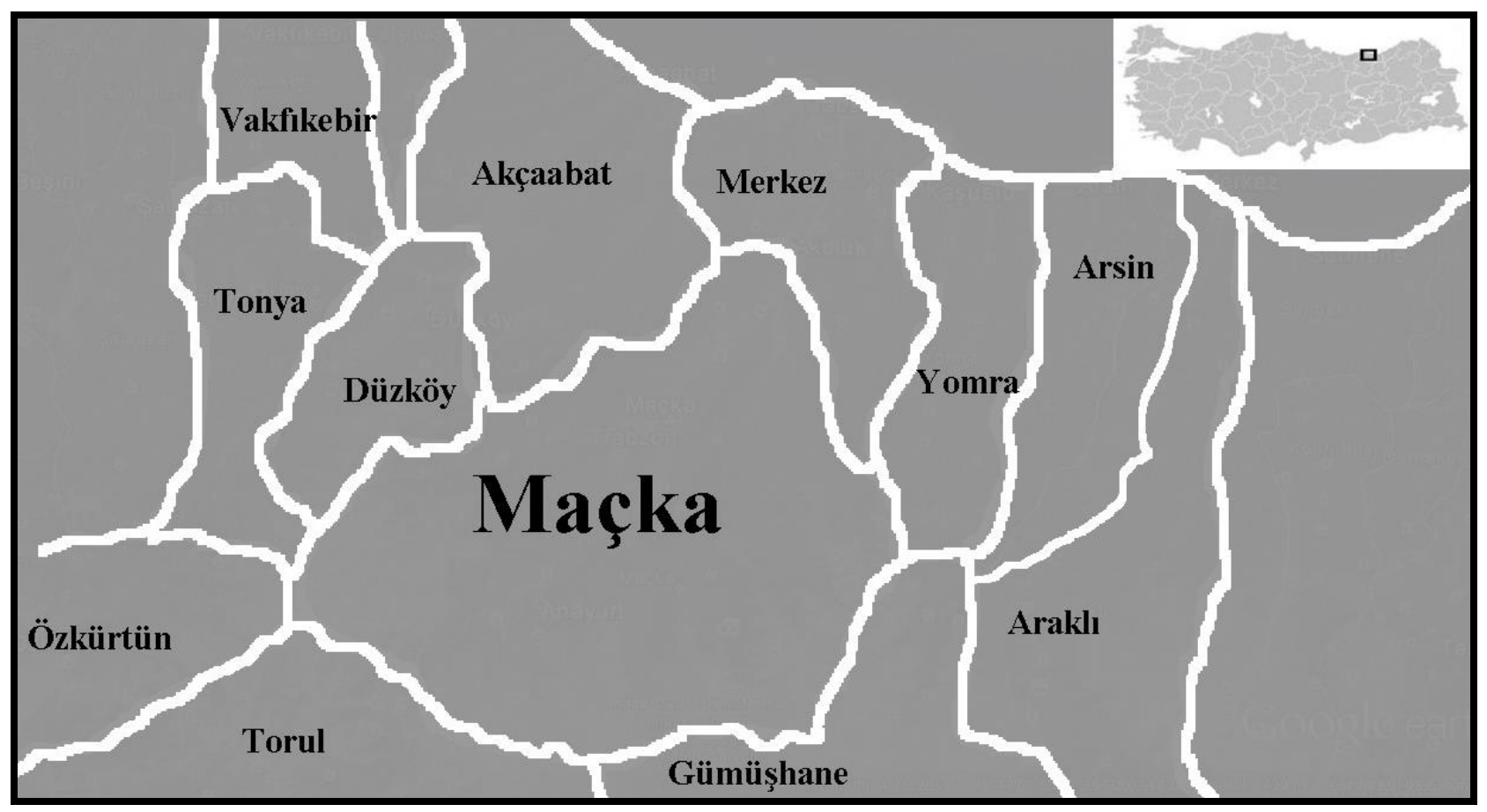

Şekil 1. Araştırma alanının haritası

Bunların yanı sıra çalışma yapılan istasyonlarla ilgili gerekli koordinat bilgileri ve ekolojik notlar arazi defterine not edilmiş. Laboratuvara getirilen örnekler öncelikle familya düzeyinde teşhisleri yapılarak ayıklanmıştır. Örnekler, literatürler ve müze materyalleri kullanılarak cins ve tür seviyesinde tespit edilerek içinde \% 70'lik alkol ve 1-2 damla gliserin bulunan saklama şişelerine konularak Gümüşhane Üniversitesi Şiran Meslek Yüksekokulu Araknoloji
Laboratuvarında (GUSAL) müze materyali olarak muhafaza altına alınmıştır.

"İncelenen örnekler" kisminda kodlama sistemi kullanılmıștır. Bu kodlamada ilk harf örneğin toplandığı il, sonraki ikinci harf ilçenin baş harfi, rakam ise örneklemenin yapıldı $\breve{g}_{1}$ yeri, küçük harf ise toplandığı tarihi göstermektedir. Örneklerin toplandığ 1 tarih ve yer bilgileri Tablo 1'de verilmiştir.

Tablo 1. Arazi çalışmasında yapılan örneklemelere ait yer bilgileri

\begin{tabular}{|c|c|c|c|c|}
\hline Kisaltmalar & Lokalite & Koordinatlar & Yükseklik (m) & Toplanma tarihi \\
\hline TMA-01 & $\begin{array}{l}\text { Trabzon, Maçka, } \\
\text { Pervanoğlu tesisi karș1s1 }\end{array}$ & $\begin{array}{l}\text { N4040'190", } \\
\text { E39 } 25^{\prime} 132^{\prime \prime}\end{array}$ & 1691 & $\begin{array}{l}\text { a) } 06.07 .2013 ; \text { b)20.07.2013; c)15.08.2013; } \\
\text { d) 22.08.2013; e)11.09.2013 }\end{array}$ \\
\hline TMA-02 & $\begin{array}{l}\text { Trabzon, Maçka, Zitaş } \\
\text { yolu }\end{array}$ & $\begin{array}{l}\mathrm{N} 40^{\circ} 20^{\prime} 570^{\prime \prime}, \\
\mathrm{E} 39^{\circ} 18^{\prime} 592^{\prime \prime}\end{array}$ & 1457 & a) 06.07 .2013 ; b) 22.08 .2013 \\
\hline TMA-03 & $\begin{array}{l}\text { Trabzon, Maçka, } \\
\text { Hamsiköy üstü }\end{array}$ & $\begin{array}{l}\mathrm{N} 40^{\circ} 22^{\prime} 199^{\prime \prime}, \\
\mathrm{E} 39^{\circ} 50^{\prime} 430^{\prime \prime}\end{array}$ & 1922 & a) 06.07 .2013 \\
\hline TMA-04 & $\begin{array}{l}\text { Trabzon, Maçka, } \\
\text { Zigana dağ }\end{array}$ & $\begin{array}{l}\text { N4039'426", } \\
\text { E39 } 24^{\prime} 710^{\prime \prime}\end{array}$ & 1723 & a) 20.07 .2013 \\
\hline TMA-05 & $\begin{array}{l}\text { Trabzon, Maçka, } \\
\text { Derebaşı yaylası }\end{array}$ & $\begin{array}{l}\mathrm{N} 40^{\circ} 39^{\prime} 107^{\prime \prime}, \\
\mathrm{E} 39^{\circ} 26^{\prime} 125^{\prime \prime}\end{array}$ & 1866 & a) 20.07 .2013 ; b) 15.08 .2013 \\
\hline TMA-06 & Trabzon, Maçka, Zitaş & $\begin{array}{l}\text { N404ㅇ' } 226^{\prime \prime}, \\
\text { E39०26'322" }\end{array}$ & 1667 & a) 20.07.2013 \\
\hline TMA-07 & $\begin{array}{l}\text { Trabzon, Maçka, Tarihi } \\
\text { eski yol-I }\end{array}$ & $\begin{array}{l}\mathrm{N} 40^{\circ} 40^{\prime} 965^{\prime \prime}, \\
\mathrm{E} 39^{\circ} 27^{\prime} 181^{\prime \prime}\end{array}$ & 1456 & a) 20.07.2013; b)29.07.2013; c)15.08.2013 \\
\hline TMA-08 & $\begin{array}{l}\text { Trabzon, Maçka, } \\
\text { Hamsiköy, Mezralar }\end{array}$ & $\begin{array}{l}\mathrm{N} 40^{\circ} 41^{\prime} 468^{\prime \prime}, \\
\mathrm{E} 39^{\circ} 27^{\prime} 979^{\prime \prime}\end{array}$ & 1428 & a) 20.07 .2013 \\
\hline
\end{tabular}




\begin{tabular}{|c|c|c|c|c|}
\hline Kisaltmalar & Lokalite & Koordinatlar & Yükseklik (m) & Toplanma tarihi \\
\hline TMA-09 & $\begin{array}{l}\text { Trabzon, Maçka, Pilav } \\
\text { dağı }\end{array}$ & $\begin{array}{l}\mathrm{N} 40^{\circ} 48^{\prime} 565^{\prime \prime} \\
\mathrm{E} 39^{\circ} 39^{\prime} 717^{\prime \prime}\end{array}$ & 1030 & $\begin{array}{l}\text { a) 17.06.2013; b) 17.07.2013; c) 21.07.2013; } \\
\text { d) } 13.09 .2013\end{array}$ \\
\hline TMA-10 & $\begin{array}{l}\text { Trabzon, Maçka, } \\
\text { Mataracılar köyü }\end{array}$ & $\begin{array}{l}\text { N4048'798", } \\
\text { E39³9'615" }\end{array}$ & 1052 & a) 21.07.2013; b)29.07.2013 \\
\hline TMA-11 & $\begin{array}{l}\text { Trabzon, Maçka, } \\
\text { Altındere vadisi }\end{array}$ & $\begin{array}{l}\text { N40³9'856", } \\
\text { E3940'108" }\end{array}$ & 1526 & a) 22.07 .2013 \\
\hline TMA-12 & $\begin{array}{l}\text { Trabzon, Maçka, } \\
\text { Sümela Manastırı }\end{array}$ & $\begin{array}{l}\mathrm{N} 40^{\circ} 41^{\prime} 600 " \\
\mathrm{E} 39^{\circ} 38^{\prime} 820^{\prime \prime}\end{array}$ & 1494 & a) 22.07 .2013 \\
\hline TMA-13 & $\begin{array}{l}\text { Trabzon, Maçka, } \\
\text { Pervanoğlu yaylası }\end{array}$ & $\begin{array}{l}\text { N4040'582", } \\
\text { E39 } 24 ' 276^{\prime \prime}\end{array}$ & 1845 & a) 28.07 .2013 \\
\hline TMA-14 & $\begin{array}{l}\text { Trabzon, Maçka, } \\
\text { Giresunda yaylası }\end{array}$ & $\begin{array}{l}\text { N40ํ1' } 716^{\prime \prime} \\
\text { E39²4'072" }\end{array}$ & 2082 & a) 28.07 .2013 ; b)11.09.2013 \\
\hline TMA-15 & $\begin{array}{l}\text { Trabzon, Maçka, } \\
\text { Balehor yaylası }\end{array}$ & $\begin{array}{l}\mathrm{N} 40^{\circ} 39^{\prime} 866^{\prime \prime} \\
\mathrm{E} 39^{\circ} 26^{\prime} 410^{\prime \prime}\end{array}$ & 1761 & a) 28.07.2013; b)22.08.2013 \\
\hline TMA-16 & $\begin{array}{l}\text { Trabzon, Maçka, Hasan } \\
\text { yaylası }\end{array}$ & $\begin{array}{l}\text { N40³9'372", } \\
\text { E39³0' 838" }\end{array}$ & 1800 & a) 28.07 .2013 \\
\hline TMA-17 & $\begin{array}{l}\text { Trabzon, Maçka, } \\
\text { Yüzüncüyıl köyü }\end{array}$ & $\begin{array}{l}\mathrm{N} 40^{\circ} 44^{\prime} 714 \\
\mathrm{E} 39^{\circ} 41^{\prime} 716^{\prime \prime}\end{array}$ & 900 & a) 29.07.2013; b)22.08.2013; c)12.09.2013 \\
\hline TMA-18 & $\begin{array}{l}\text { Trabzon, Maçka, } \\
\text { Çayırlar köyü }\end{array}$ & $\begin{array}{l}\text { N4046'241", } \\
\text { E3940'732" }\end{array}$ & 735 & a) 29.07 .2013 \\
\hline TMA-19 & $\begin{array}{l}\text { Trabzon, Maçka, } \\
\text { Camiboğazı yaylası }\end{array}$ & $\begin{array}{l}\text { N4036'119", } \\
\text { E39³8' 776" }\end{array}$ & 2448 & a) 30.07 .2013 \\
\hline TMA-20 & $\begin{array}{l}\text { Trabzon, Maçka, } \\
\text { Çakırgöl yolu }\end{array}$ & $\begin{array}{l}\text { N4038'654", } \\
\text { E3940' 843" }\end{array}$ & 1841 & a) 30.07 .2013 \\
\hline TMA-21 & $\begin{array}{l}\text { Trabzon, Maçka, Tarihi } \\
\text { eski yol-II }\end{array}$ & $\begin{array}{l}\text { N4040'972", } \\
\text { E39²7'210" }\end{array}$ & 1577 & a) 15.08 .2013 \\
\hline TMA-22 & $\begin{array}{l}\text { Trabzon, Maçka, Tarihi } \\
\text { eski yol-III }\end{array}$ & $\begin{array}{l}\text { N404' } 630^{\prime \prime} \\
\mathrm{E} 39^{\circ} 28^{\prime} 740^{\prime \prime}\end{array}$ & 1323 & a) 15.08 .2013 \\
\hline TMA-23 & $\begin{array}{l}\text { Trabzon, Maçka, } \\
\text { Örnekalan köyü }\end{array}$ & $\begin{array}{l}\mathrm{N} 40^{\circ} 50^{\prime} 403^{\prime \prime} \\
\mathrm{E} 39^{\circ} 36^{\prime} 112^{\prime \prime}\end{array}$ & 940 & a) 16.08 .2013 \\
\hline TMA-24 & $\begin{array}{l}\text { Trabzon, Maçka, Kulin } \\
\text { dağ1 yol ayrımı }\end{array}$ & $\begin{array}{l}\mathrm{N} 40^{\circ} 50^{\prime} 130^{\prime \prime} \\
\mathrm{E} 39^{\circ} 34^{\prime} 525^{\prime \prime}\end{array}$ & 1482 & a) 16.08 .2013 \\
\hline TMA-25 & $\begin{array}{l}\text { Trabzon, Maçka, Ocaklı } \\
\text { köyü yaylası }\end{array}$ & $\begin{array}{l}\text { N4049'551", } \\
\text { E39²9'191" }\end{array}$ & 1910 & a) 16.08 .2013 \\
\hline TMA-26 & $\begin{array}{l}\text { Trabzon, Maçka, Lişer } \\
\text { yaylası }\end{array}$ & $\begin{array}{l}\text { N4049'500", } \\
\text { E39²9'940" }\end{array}$ & 1917 & a) 16.08 .2013 ; b)14.09.2013 \\
\hline TMA-27 & $\begin{array}{l}\text { Trabzon, Maçka, Kulin } \\
\text { dağı }\end{array}$ & $\begin{array}{l}\text { N4049'190", } \\
\text { E39³4'227" }\end{array}$ & 1061 & a) 16.08 .2013 ; b)14.09.2013 \\
\hline TMA-28 & $\begin{array}{l}\text { Trabzon, Maçka, } \\
\text { Yukarı köy }\end{array}$ & $\begin{array}{l}\text { N4045'045", } \\
\text { E39 } 34^{\circ} 070^{\prime \prime}\end{array}$ & 691 & a) 17.08 .2013 \\
\hline TMA-29 & $\begin{array}{l}\text { Trabzon, Maçka, } \\
\text { Yazılıtaş köy }\end{array}$ & $\begin{array}{l}\text { N4044'221", } \\
\text { E39³3'085" }\end{array}$ & 837 & a) 17.08 .2013 \\
\hline TMA-30 & $\begin{array}{l}\text { Trabzon, Maçka, } \\
\text { Dikkaya köyü }\end{array}$ & $\begin{array}{l}\text { N4042'012", } \\
\text { E39 } 29^{\prime} 395^{\prime \prime}\end{array}$ & 1045 & a) 17.08 .2013 \\
\hline TMA-31 & $\begin{array}{l}\text { Trabzon, Maçka, } \\
\text { Papazar yaylası }\end{array}$ & $\begin{array}{l}\text { N404ㄴ' } 747^{\prime \prime}, \\
\text { E39²8'572" }\end{array}$ & 1798 & a) 17.08 .2013 \\
\hline TMA-32 & $\begin{array}{l}\text { Trabzon, Maçka, } \\
\text { Yediharman yaylası }\end{array}$ & $\begin{array}{l}\mathrm{N} 40^{\circ} 40^{\prime} 675^{\prime \prime} \\
\mathrm{E} 39^{\circ} 25^{\prime} 657^{\prime \prime}\end{array}$ & 1385 & a) 22.08 .2013 \\
\hline TMA-33 & $\begin{array}{l}\text { Trabzon, Maçka, } \\
\text { Gıranobası yaylası }\end{array}$ & $\begin{array}{l}\text { N4041'269", } \\
\text { E39²7'953" }\end{array}$ & 1626 & a) $22.08 .2013 ;$ b)12.09.2013 \\
\hline TMA-34 & $\begin{array}{l}\text { Trabzon, Maçka, } \\
\text { Galyan yaylası }\end{array}$ & $\begin{array}{l}\text { N4041'929", } \\
\text { E3943'737" }\end{array}$ & 1953 & a) 23.08 .2013 \\
\hline TMA-35 & $\begin{array}{l}\text { Trabzon, Maçka, } \\
\text { Büyükoba yaylası }\end{array}$ & $\begin{array}{l}\mathrm{N} 40^{\circ} 42^{\prime} 214^{\prime \prime}, \\
\mathrm{E} 39^{\circ} 19^{\prime} 885^{\prime \prime}\end{array}$ & 2263 & a) 12.09 .2013 \\
\hline TMA-36 & $\begin{array}{l}\text { Trabzon, Maçka, } \\
\text { Güzelyayla köyü }\end{array}$ & $\begin{array}{l}\text { N4041'259", } \\
\text { E39०29'199" }\end{array}$ & 1098 & a) 12.09 .2013 \\
\hline TMA-37 & $\begin{array}{l}\text { Trabzon, Maçka, } \\
\text { Çataltepe șehitliği }\end{array}$ & $\begin{array}{l}\text { N4042'673", } \\
\text { E39 } 43^{\circ} 867^{\prime \prime}\end{array}$ & 1497 & a) 13.09 .2013 \\
\hline TMA-38 & $\begin{array}{l}\text { Trabzon, Maçka, } \\
\text { Karadağ }\end{array}$ & $\begin{array}{l}\text { N4042'842", } \\
\text { E39 } 43^{\prime} 200^{\prime \prime}\end{array}$ & 1670 & a) 13.09 .2013 \\
\hline TMA-39 & $\begin{array}{l}\text { Trabzon, Maçka, Pilav } \\
\text { dağı-II }\end{array}$ & $\begin{array}{l}\text { N4049'299", } \\
\text { E39³9'818" }\end{array}$ & 1140 & a) 13.09 .2013 \\
\hline TMA-40 & $\begin{array}{l}\text { Trabzon, Maçka, } \\
\text { Ormaniçi köyü }\end{array}$ & $\begin{array}{l}\mathrm{N} 40^{\circ} 46^{\prime} 48,4^{\prime \prime} \\
\mathrm{E} 39^{\circ} 40^{\prime} 36,8^{\prime \prime}\end{array}$ & 950 & a) 13.09 .2013 \\
\hline
\end{tabular}




\section{Bulgular}

Araştırma alanı olan Maçka ilçesi ve çevresinde yapılan arazi çalışmalarında örnekler el, atrap, çukur tuzak yardımıyla toplandi. Toplanan örnekler faunistik ve sistematik açıdan değerlendirildi. $\mathrm{Bu}$ değerlendirme sonucunda 4 familya ait toplam 19 takson tespit edilmiştir. Tespit edilen taksonların listesi Tablo 2'de verilmiştir.
Dicranolasmatidae Simon, 1879

Dicranolasma giljarovi Šilhavý, 1966

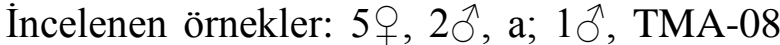
a; 1, 1ठ, TMA-30 a; 1q, 3ð, TMA-10 a. Dünyadaki yayılışı: Bulgaristan, Gürcistan, Kırım, Rodos Adaları, Rusya, Türkiye ve Ukrayna (Snegovaya ve Marusik, 2012). Türkiye'deki yayılış1: Ankara, Van (Kurt vd., 2010), Kastamonu (Snegovaya ve Marusik 2012), Gümüşhane (Kurt ve Erman, 2015).

Tablo 2. Araştırma alanından tespit edilen otbiçen taksonlarının listesi.

\begin{tabular}{|c|c|}
\hline Familya/Türler & Cinsiyet \\
\hline \multicolumn{2}{|l|}{ Dicranolasmatidae } \\
\hline Dicranolasma giljarovi Šilhavý, 1966 & q, $0^{\lambda}$ \\
\hline \multicolumn{2}{|l|}{ Nemastomatidae } \\
\hline Giljarovia tenebricosa Redikorzev, 1936 & q, $0^{\lambda}$ \\
\hline Histricostoma caucasicum (Redikorzev, 1936) & q, $0^{-1}$ \\
\hline Mitostoma gracile (Redikorzev, 1936) & q, $0^{\lambda}$ \\
\hline Paranemastoma sp. & q, $0^{\lambda}$ \\
\hline Vestiferum alatum Martens, 2006 & $\hat{0}$ \\
\hline Vestiferum funebre (Redikorzev, 1936) & q, $0^{\lambda}$ \\
\hline \multicolumn{2}{|l|}{ Phalangiidae } \\
\hline Lacinius erinaceus Staręga, 1966 & q, $0^{\lambda}$ \\
\hline Mitopus morio (Fabricius, 1779) & q, $\hat{O}^{-1}$ \\
\hline Odiellus zecariensis Mcheidze 1952 & q, $0^{\lambda}$ \\
\hline Opilio hemseni Roewer, 1952 & q, $\hat{O}^{-1}$ \\
\hline Opilio parietinus (De Geer, 1778) & q, $0^{\lambda}$ \\
\hline Phalangium opilio Linnaeus, 1761 & o, $\hat{\sigma}^{\lambda}$ \\
\hline Rafalskia olympica olympica (Kulczyński, 1903) & q, $\hat{O}^{-1}$ \\
\hline Rilaena ermani Kurt, 2015 & q, $0^{\lambda}$ \\
\hline Rilaena triangularis (Herbst, 1799) & q \\
\hline Rilaena zakatalica Snegovaya, Chemeris, 2005 & $\sigma^{\lambda}$ \\
\hline Zachaeus crista (Brullé, 1832) & q, $0^{\lambda}$ \\
\hline \multicolumn{2}{|l|}{ Sclerosomatidae Simon, 1879} \\
\hline Nelima pontica Charitonov, 1941 & q, $\hat{O}^{-1}$ \\
\hline
\end{tabular}


Nemastomatidae Simon, 1872

Giljarovia tenebricosa (Redikorzev, 1936)

İncelenen örnekler: $1 \hat{\jmath}$, TMA-09 a; 1 +, $1 \hat{0}$, TMA-01 a; 3ㅇ, 2ภ, TMA-09 c; 1q, 1§,

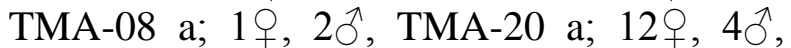
TMA-26 b; 1 ㅇ, 1ठ, TMA-31 a; $1 \hat{\jmath}$, TMA-01

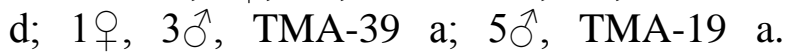
Dünyadaki yayılış1: Abhazya, Azerbaycan, Gürcistan ve Türkiye (Martens, 2006). Türkiye'deki yayılışı: Artvin, Rize (Martens, 2006), Gümüşhane (Kurt ve Erman, 2015).

Histricostoma caucasicum (Redikorzev, 1936)

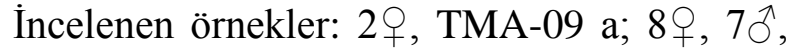

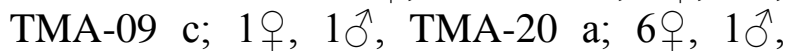
TMA-17 c; $1 \delta^{\lambda}$, TMA-27 a; 2ᄋ, TMA-31 a; 1§, TMA-39 a; 2へ, TMA-40 a; $3+$, TMA12; $2 \hat{\jmath}$, TMA-24 a; 4, 3ð, TMA-37 a; 2 , TMA-10 a; 3q, 2ð,TMA-02 a. Dünyadaki yayılışı: Azerbaycan, Ermenistan, Gürcistan ve Türkiye (Martens, 2006). Türkiye'deki yayılışı: Artvin, Ordu, Rize (Martens, 2006), Gümüşhane (Kurt ve Erman, 2015).

Mitostoma gracile (Redikorzev, 1936)

İncelenen örnekler: $2+, 2 \hat{\gamma}$, TMA-09 a; 1 ,

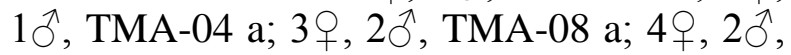
TMA-12 a; 5ㅇ, 6ㅅ, TMA-27 a; $2+, 3 \hat{\jmath}$, TMA-09 c. Dünyadaki yayılışı: Azerbaycan, Bulgaristan, Gürcistan, Rusya, Türkiye ve Yunanistan (Martens, 2006). Türkiye'deki yayılışı: Kastamonu, Ordu, Rize (Martens, 2006), Gümüşhane (Kurt ve Erman, 2015).

\section{Paranemastoma sp.}

İncelenen örnekler: $2+, 2 \hat{q}$, TMA-09 a; $1+$,

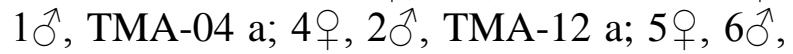
TMA-27 a; 2 +, $3 \hat{\jmath}$, TMA-09 c.

Vestiferum alatum Martens, 2006

İncelenen örnekler: 3 $\hat{\partial}, \quad$ TMA-09 d. Dünyadaki yayılış1: Gürcistan ve Türkiye (Martens, 2006). Türkiye'deki yayılıșı: Artvin (Martens, 2006), Trabzon (Kurt, 2015b).

Vestiferum funebre (Redikorzev, 1936)

İncelenen örnekler: 2ㅇ, 10ิ, TMA-09 c.

Dünyadaki yayılışı: Gürcistan ve Türkiye (Martens, 2006; Kurt, 2015b). Türkiye'deki yayılış1: Trabzon (Kurt, 2015b).
Phalangiidae Latreille, 1802

Lacinius erinaceus Staręga, 1966

İncelenen örnekler: 29, TMA-15 a; 2へ, TMA-15 b. Dünyadaki yayılışı: Abhazya (Staręga 1966) ve Türkiye (Kurt ve Erman, 2015). Türkiye'deki yayılışı: Bayburt, Gümüşhane (Kurt ve Erman, 2015).

Mitopus morio (Fabricius, 1779)

İncelenen örnekler: 5 ㅇ, $3 \hat{\jmath}$, TMA-20 a; 1 ,

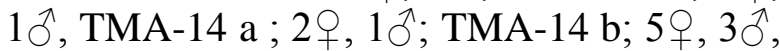
TMA-13 a; 2へ, TMA-17 c; 5 , TMA-26 a;

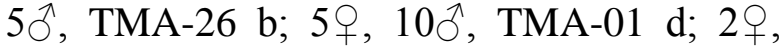
2へ, TMA-07 a; 1 ㅇ, $1 \hat{\jmath}$, TMA-38 a; $5+, 5 \hat{\jmath}$,

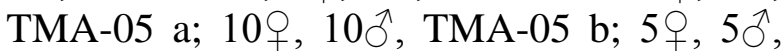
TMA-01 c; 3ㅇ, 1ð, TMA-33 a; 5ㅇ, $1 \hat{\jmath}$, TMA-33 b; 10, TMA-27 a. Dünyadaki yayılışı: Almanya, Arnavutluk, Avusturya, Belçika, Bosna-Hersek, Bulgaristan, Çek Cumhuriyeti, Çin, Danimarka, Estonya, Fareo Adaları, Finlandiya, Fransa, Himalayalar, Hirvatistan, Hollanda, Japonya, İran, İspanya, İsveç, İsviçre, İtalya, İzlanda, Kore, Kuzey Afrika, Kuzey Amerika, Macaristan, Norveç, Polonya, Romanya, Rusya, Sibirya, Slovakya, Slovenya, Tibet ve Türkiye (Spoek, 1963). Türkiye'deki yayılışı: Mardin (Roewer, 1959), Niğde (Kurt, vd., 2008), Bayburt (Kurt ve Erman, 2015).

Odiellus zecariensis Mcheidze 1952

İncelenen örnekler: 1ठ, TMA- $40 \mathrm{a} ; 1 \hat{\jmath}$,

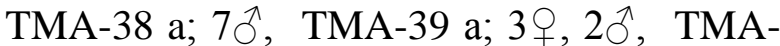
09 a ; 19, 10, TMA-09 b; 1, 1ڤ, TMA-09

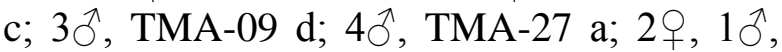
TMA-02 b; 10 , TMA-17 a; $3 \hat{\delta}$, TMA-24 a;

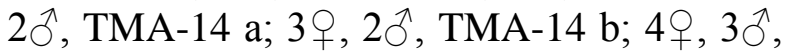
TMA-36 a; 3 , , 4今, TMA-26 a. Dünyadaki yayılışı: Abhazya, Ajaria, Kırım, Gürcistan, Rusya ve Türkiye (Snegovayave Marusik, 2012). Türkiye'deki yayılışı: Artvin (Snegovaya ve Marusik, 2012).

Opilio hemseni Roewer, 1952

İncelenen örnekler: 1 ,, $4 \hat{\jmath}$, TMA-40 a; $5 \hat{\jmath}$, TMA-36 a; 2ᄋ, TMA-07 a; 2へ, TMA-07 b; 3 , $3 \hat{\jmath}$, TMA-22 a; 5q, TMA-33 a; $3 \hat{\jmath}$, TMA-33 a; 5, TMA-21 a; 1ठิ, TMA-39 a. Dünyadaki yayılışı: Ermenistan, Gürcistan, İran, Rusya, Türkiye ve Ukrayna (Staręga, 
2003; Snegovaya, 2010). Türkiye'deki yayılışı: Artvin (Staręga, 2003).

Opilio parietinus (De Geer, 1778)

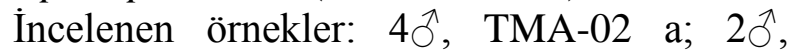

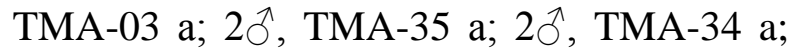
3 , $2 \hat{\jmath}$, TMA-33 a; $39,4 \hat{\jmath}$, TMA-30 a; 5 ,

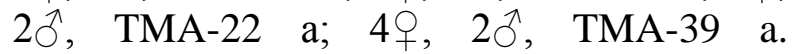
Dünyadaki yayılışı: A.B.D., Avrupa'nın tamamı, Batı Sibirya, Kafkaslar, Kanada, Kanarya Adaları, Kuzey Afrika, Merkezi Asya, Tasmanya ve Yakın Doğu (Mitov, 2000). Türkiye'deki yayıllışı: Adana, Ankara, Kastamonu, Kayseri, Kırıkkale, Kırşehir, Konya, Niğde, Osmaniye (Kurt vd., 2011), Antalya (Çorak, 2010), Bayburt, Gümüşhane (Kurt ve Erman, 2015),

\section{Phalangium opilio Linnaeus, 1761}

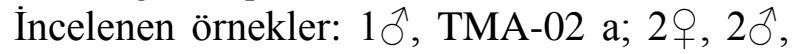
TMA-09 a; 4, 1ठ, TMA-09 b; 2ڤ, TMA-09 c; 2早, TMA-09 d; 10 +, $10 \hat{\jmath}$, TMA-13 a; 10, 10^, TMA-17 a; 5ㅇ, 5ภ, TMA-17 b;

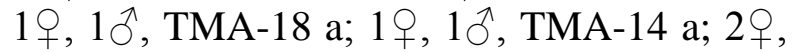

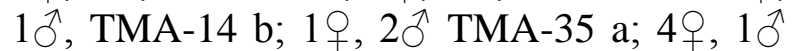
TMA-07 a; 3ㅇ, 1ठ, TMA-21 a; 2 , , $1 \hat{0}$, TMA-22 b; 10ㅇ, 5ภ, TMA-39 a; 10 +, $5 \hat{\jmath}$,

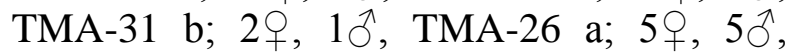
TMA-26 b; 5 , $1 \hat{\jmath}$, TMA-01 a; $1 \hat{\jmath}$, TMA-01 b; 2ᄋ, 1ڤ, TMA-01 c; 5, 2犬, TMA-06 a; 3 , $2 \hat{\circ}$, TMA-15 a; 2 , TMA-33 a; 4 , TMA-33 b. Dünyadaki yayılışı: Almanya, Arnavutluk, Avustralya, Belçika, BosnaHersek, Bulgaristan, Çek Cumhuriyeti, Danimarka, Finlandiya, Hollanda, Japonya, İspanya, İsveç, İsviçre, İtalya, Kuzey Amerika, Macaristan, Norveç, Polonya, Rusya, Slovakya, Slovenya, Türkiye, Yeni Zelanda ve Yugoslavya (Spoek, 1963).

Türkiye'deki yayılışı: Ankara, Hatay, Kırıkkale, Niğde (Kurt vd., 2010), Bayburt, Gümüşhane (Kurt ve Erman, 2015).

Rafalskia olympica olympica (Kulczyński, 1903)

İncelenen örnekler: 10̂, TMA-01 c. Dünyadaki yayılışı: Türkiye (Karaman, 2002). Türkiye'deki yayılışı: Bursa (Karaman, 2002).

Rilaena ermani Kurt, 2015
İncelenen örnekler: 1ð, TMA-01 a, 2q, TMA-01 b, 29, TMA-01 c. Dünyadaki yayılışı: Türkiye (Kurt, 2015a). Türkiye'deki yayılış1: Trabzon (Kurt, 2015a).

Rilaena triangularis (Herbst, 1799)

İncelenen örnekler: 2우 TMA-05 a; 19, TMA-20 a; 19, TMA-15 a. Dünyadaki yayılışı: A.B.D., Almanya, Avusturya, Belçika, Bosna-Hersek, Bulgaristan, Büyük Britanya, Çek Cumhuriyeti, Danimarka, Finlandiya, Fransa, Hirvatistan, Hollanda, İrlanda, İsveç, İsviçre, İzlanda, İtalya, Kanada, Kolombiya, Macaristan, Norveç, Polonya, Romanya, Rusya, Surbistan, Slovakya ve Slovenya (Martens, 1978). Türkiye'deki yayılışı: Bayburt (Kurt ve Erman, 2015).

Rilaena zakatalica Snegovaya, Chemeris, 2005

İncelenen örnekler: $1 \hat{\jmath}, \quad$ TMA-01 c. Dünyadaki yayılışı: Azerbaycan, Rusya, Türkiye (Snegovaya ve Chemeris 2005, Snegovaya ve Chumachenko 2011; Kurt, 2015a). Türkiye'deki yayılışı: Trabzon (Kurt 2015a).

Zachaeus crista (Brullé, 1832)

İncelenen örnekler: $5 \hat{\jmath}, 5 q$, TMA-04 a; $5 q$, $5 \widehat{\jmath}$, TMA-20 a; 2 , TMA-24 a; 3 , TMA-32 a. Dünyadaki yayılışı: Almanya, Azerbaycan, Avusturya, Belçika, Çek Cumhuriyeti, Danimarka, Hollanda, İsviçre, İsveç, Macaristan, Norveç, Polonya, Slovakya, Slovenya ve Türkiye (Snegovaya ve Marusik, 2012). Türkiye'deki yayılış1: Ankara, Bolu, Kırıkkale, Niğde, Osmaniye (Kurt, vd., 2011), Antalya (Çorak, 2010), Bilecik, Denizli (Martens, 1978), Bursa, İzmir (Snegovaya ve Marusik, 2012), Bayburt, Gümüşhane (Kurt ve Erman, 2015).

Sclerosomatidae Simon, 1879

Nelima pontica Charitonov, 1941

İncelenen örnekler: 1今, TMA-18 a; 20ㅇ, 20 $\hat{\circ}$, TMA-03 a; 10 ㅇ, $10 \hat{\jmath}$, TMA-36 a; $10 \hat{\partial}$,

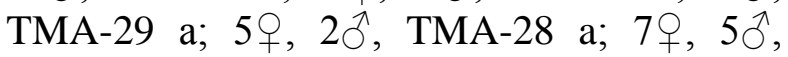

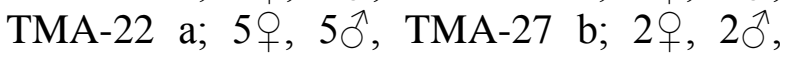
TMA-40 a; 10 +, $5 \AA$, TMA-07 a; 2 + , $1{ }^{\lambda}$, 
TMA-30 a; 1ㅇ, 20ิ TMA-09 a. Dünyadaki yayılış1: Abhazya, Bulgaristan, Gürcistan, Rusya ve Türkiye (Snegovayave Marusik, 2012). Türkiye'deki yayılışı: Kastamonu, Kurklareli (Kurt, 2014).

\section{Tartışma ve Sonuç}

Bu çalışma Maçka (Trabzon) ilçesinin otbiçen faunasını belirlemek amacıyla yapılmıştır. Araştırma alanında yapılan arazi çalışmalarında örnekler el, atrap, çukur tuzak yardımıyla toplandi. Toplanan örnekler faunistik ve sistematik açıdan değerlendirildi. $\mathrm{Bu}$ değerlendirme sonucunda 4 familya ait toplam 19 takson tespit edilmiştir. $\mathrm{Bu}$ taksonlar: Dicranolasma giljarovi Šilhavý, 1966; Giljarovia tenebricosa Redikorzev, 1936; Histricostoma caucasicum (Redikorzev, 1936); Mitostoma gracile (Redikorzev, 1936); Paranemastoma sp.; Vestiferum alatum Martens, 2006; Vestiferum funebre (Redikorzev, 1936); Lacinius erinaceus Staręga, 1966; Mitopus morio (Fabricius, 1779); Odiellus zecariensis Mcheidze 1952; Opilio hemseni Roewer, 1952; Opilio parietinus (De Geer, 1778); Phalangium opilio Linnaeus, 1761; Rafalskia olympica olympica (Kulczyński, 1903); Rilaena ermani Kurt, 2015; Rilaena zakatalica Snegovaya, Chemeris, 2005; Rilaena triangularis (Herbst, 1799); Zachaeus crista (Brullé, 1832); Nelima pontica Charitonov, 1941 taksonları araştırma alanından ilk kez kaydedilmiştir.

Arazi çalışmaları sonucunda toplam 1000 birey yakalanmış olup bunların 347'si erkek, 375'i dişi ve 278'i nimftir. Değerlendirilen örneklerden 424 birey Phalangiidae, 159 birey Nemastomatidae, 125 birey Sclerosomatidae, 14 birey ise Dicranolasmatidae familyasina ait olduğu tespit edilmiştir.

Çalışma alanının otbiçen faunası Kafkasların otbiçen faunasına benzerlik gösterdiği tespit edilmiştir. Bunun nedeni Kafkasya'ya ait fauna elemanlarının ülkemize giriş kapısı Kars-Erzurum Platosu ve Doğu Karadeniz kıyı şeridi olmasıdır.
Birçok Avrupa ülkesi 19. yüzyılda otbiçen faunalarını tespit etmelerine rağmen, ülkemizde ise henüz otbiçen faunası detaylı olarak belirlenememiştir. Buna bağlı olarak, mevcut çalışma gibi faunistik ve sistematik çalışmaların daha çok proje konusu olarak verilmesi ve daha kapsamlı çalışmalar yapılması, ülkemizdeki otbiçen faunasına katkı sağlayacaktır.

\section{Teşekkür}

Türkiyenin opilionid zenginliğini ortaya çıkarmak ve katkılar sağlamak amacıyla yapılan bu çalışmaya 13.B0423.02.2 nolu proje ile maddi destek veren Gümüşhane Üniversitesi Bilimsel Araştırma Projeleri Birimine (GÜBAP) teşekkürü borç bilirim.

\section{Kaynaklar}

Bayram, A., Çorak, İ. ve Danışman, T., 2007. Ankara-Soğuksu Milli Parkı ve çevresinin otbiçen faunasının araştırılması (Arachnida: Opiliones). Tübitak, Tbag, No: 2437/104T046, Ankara.

Çorak, İ., 2010. Antalya ili Otbiçenlerin Sistematiği ve Biyoekolojisi (Arachnida: Opiliones). Doktora Tezi, Kırıkkale Üniversitesi Fen Bilimleri Enstitüsü. Kırıkkale, 131s.

Hillyard, P.D. ve Sankey, J.H.P., 1990. Harvestmen: Keys and notes for the identification of the species. Synopses of the British Fauna (Linnean Society of London), London, England. pp. 1-120.

Karaman, I.M., 2002. A contribution to the knowledge of the species Rafalskia olympica (Kulczyński, 1903) (Opiliones, Phalangiidae, Phalangiinae). Arachnologische Mitteilungen, 24, 62-71.

Kurt, K., Babaşoğlu, A., Seyyar, O., Demir, H. ve Topçu, A., 2008. New faunistic records for the Turkish harvestmen fauna (Arachnida: 
Opiliones). Munis Entomology \& Zoology, 3(2), 654-660.

Kurt, K., 2010. Gümüşhane ve Bayburt illerinin otbiçen faunasi. Doktora Tezi, Atatürk Üniversitesi Fen Bilimleri Enstitüsü. Erzurum, 147s.

Kurt, K., Erman, O.K., Demir, H. ve Seyyar, O., 2010. The Turkish Harvestmen (Opiliones) with zoogeographical remarks. Serket, 12 (2), 33-44.

Kurt, K., Snegovaya, N., Demir, H. ve Seyyar, O., 2011. New Data on the Harvestmen (Arachnida, Opiliones) of Turkey. Acta Zoologica Bulgarica, 63 (2), 145-150.

Kurt, K. ve Erman, Ö.K., 2015. Harvestmen (Arachnida: Opiliones) fauna of Gümüşhane and Bayburt in Turkey. Spixiana (In press).

Kurt, K., 2014. Updated checklist of harvestmen (Arachnida: Opiliones) in Turkey. Archives of Biological Sciences, Belgrade, 66, 1617-1631.

Kurt, K., 2015a. A new species and a new record of the genus Rilaena Šilhavy, 1965 (Opiliones: Phalangiidae) from Turkey. Entomological News (In Press).

Kurt, K., 2015b. Contributions to the knowledge of the genus Vestiferum Martens, 2006 (Opiliones: Nemastomatidae) from Turkey. Entomological News (In Press).

Kury, A.B., 2012. A synopsis of catalogs and checklists of harvestmen (Arachnida, Opiliones). Zootaxa, 3184, 35-58.

Kury, A. B., 2013. Order Opiliones Sundevall, 1833. In: Zhang, Z.-Q. (Ed.), Animal biodiversity: an outline of higher-level classification and survey of taxonomic richness (Addenda 2013). Zootaxa, 3703, 2733.
Martens, J., 1978. Spinnentiere, Arachnida: Weberknechte, Opiliones. Die Tierwelt Deutschlands. G. Fischer Verlag, Jena, Deutchland. pp. 1-464.

Martens, J., 2006. Weberknechte aus dem Kaukasus (Arachnida, Opiliones, Nemastomatidae) [Harvestmen from the Caucasus (Arachnida, Opiliones, Nemastomatidae)]. Senckenbergiana Biologica, 86 (2), 145-210.

Mitov, P.G., 2000. Contribution to the knowledge of the harvestmen (Arachnida: Opiliones) of Albania. Ekologia, 19 (3), 159-170.

Mitov, P.G., 2007. Spatial Niches of Opiliones (Arachnida) from Vitosha Mountains, Bulgaria. Biogeography and Ecology of Bulgaria, Monographiae Biologicae, 82, 423446.

Roewer, C.F., 1959. Die Araneae, Solifuga und Opiliones der Sammlungen des Herrn Dr. K. Lindberg aus Griechenland, Creta, Anatolien, Iran und Indien. Göteborgs Kungliga Vetenskaps- och VitterhetsSamhälles handlingar. Matematiska och naturvetenskapliga skrifter, 8 (4), 1-47.

Snegovaya, N.Y. ve Chemeris, A.N., 2005. A contribution to the knowledge of the harvestman fauna of the Zakataly State Reserve, Azerbaijan (Arachnida: Opiliones). Arthropoda Selecta, 13 (4), 263.

Snegovaya, N.Y., 2010. Further studies on harvestmen of the genus Opilio Herbst, 1798 (Arachnida: Opiliones: Phalangiidae) from the Caucasus. Caucasian Entomological Bulletin, 6 (1), 3-18.

Snegovaya, N.Y. ve Chumachenko, Y.A., 2011. Harvestmen (Arachnida: Opiliones) from the yew and boxtree grove of the Caucasian State 
Natural Biospheric Reserve, Russia. Caucasian Entomological Bulletin, 7 (2), 115-124.

Snegovaya, N.Yu., ve Marusik, Y.M., 2012. New species and collections of Opiliones (Arachnida) from Turkey. Acta Arachnologica, 61(2): 59-70.
Spoek, G.L., 1963. The Opilionida (Arachnida) of the Netherlands. Zoologische Verhandelingen, 63, 170 .

Staręga, W., 2003. On the identity and synonymies of some Asiatic Opilioninae (Opiliones: Phalangiidae). Acta Arachnologica, 52 (2), 91-102. 